\title{
Search for additional heavy scalar/pseudoscalar neutral Higgs bosons at CMS
}

\author{
Ram Krishna Dewanjee* \\ (On behalf of CMS collaboration) \\ Author affiliation \\ Tata Insitute Of Fundamental Research, Homi Bhabha Road, Colaba, Mumbai- 400005. \\ E-mail: ram.krishna.dewanjee@cern.ch
}

\begin{abstract}
Recent results on searches for additional scalar and pseudo-scalar neutral Higgs bosons are presented. These searches are well motivated within a variety of BSM models, from the MSSM to more general two Higgs doublet models. Heavy neutral scalar Higgs (H) or pseudo-scalar (A) bosons are searched in different final states. These studies are based on pp collision data collected at $\sqrt{s}=7$ and $8 \mathrm{TeV}$ by the CMS collaboration at the LHC.
\end{abstract}

XXIV International Workshop on Deep-Inelastic Scattering and Related Subjects

11-15 April, 2016

DESY Hamburg, Germany

\footnotetext{
* Speaker.
} 


\section{CMS Detector}

CMS is a general purpose particle detector [1] featuring a superconducting solenoid (6 m internal diameter), providing a magnetic field of 3.8 Tesla. Within the solenoid volume are a silicon pixel and strip tracker, a lead tungstate crystal electromagnetic calorimeter, and a brass/scintillator hadron calorimeter, each composed of a barrel and two endcap sections. Muons are measured in gas-ionization detectors embedded in the steel flux-return yoke outside the solenoid. Extensive forward calorimetry complements the coverage of the barrel and endcap detectors. Event selection is done in 2 stages (called triggers): Level-1 (40 MHz to $100 \mathrm{kHz}$ ) and High Level Trigger (HLT) $(100 \mathrm{kHz}$ to $100 \mathrm{~Hz})$. While Level-1 is predominantly hardware oriented, HLT is software based and is implemented in a farm of about 10000 commercial processor cores.

\section{Introduction}

The discovery of a scalar boson [2] and the measurement of its properties (mass, spin, CP etc.) [3] have established the Standard Model (SM) of particle physics and the Brout-Englert-Higgs mechanism. However, within the present experimental precision, the discovered boson may just as well be one member of an extended family of scalars and/or pseudo-scalars that arise as a consequence of more complex scalar sectors predicted by a variety of beyond standard model (BSM) theories. Important types of such theoretical models include supersymmetric theories (e.g. MSSM) and 2-Higgs doublet models (2HDM). These additional heavy scalar/pseudo-scalar particles will be henceforth referred to as BSM Higgs bosons.

CMS performed a thorough search of BSM Higgs bosons using full Run- 1 luminosity ${ }^{1}$ in a wide variety of theoretical scenarios and final states. Most of these searches probed BSM Higgs decays to $3^{\text {rd }}$ generation down-type fermions (b-quarks and taus). This is because most BSM theories (e.g. MSSM) predict enhancement of BSM Higgs branching fractions to them compared to the corresponding values for SM Higgs boson. In what follows, we will briefly describe the following searches:

- Search for MSSM neutral Higgs bosons $(\phi=\mathrm{h} / \mathbf{H} / \mathbf{A})^{2}$ in their decays to pairs of b-quarks $(\phi \rightarrow b \bar{b})[9]$ and tau leptons $(\phi \rightarrow \tau \bar{\tau})[8]$.

- Search for heavy neutral MSSM Higgs boson $(\mathbf{H})$ decaying to 2 SM Higgs bosons $(\mathbf{H} \rightarrow \mathrm{hh})^{3}$ one of which decays to pair of b-quarks $(h \rightarrow b \bar{b})$ and the other to a pair of tau leptons $(h \rightarrow \tau \bar{\tau})[10]$.

- Search for a heavy pseudo-scalar Higgs boson (A) decaying to a Z-boson and a SM Higgs boson $(\mathbf{A} \rightarrow \mathrm{Zh})$ wherein the former decays to light leptons $(\mathrm{Z} \rightarrow l \bar{l}$ where $l$ represents an electron or a muon) and the latter decays to either a b-quark pair $(h \rightarrow b \bar{b})[11]$ or a tau lepton pair $(h \rightarrow \tau \bar{\tau})[10]$.

\footnotetext{
${ }^{1} 20 \mathrm{fb}^{-1}$ collected at $\sqrt{(s)}=8 \mathrm{TeV}$ and $5 f b^{-1}$ collected at $\sqrt{(s)}=7 \mathrm{TeV}$.

${ }^{2}$ The MSSM Higgs sector has 2 complex scalar doublets leading to 5 physical Higgs bosons: a heavy/light CP even scalar $(\mathbf{H} / \mathrm{h})$, a pseudo-scalar $(\mathbf{A})$ and 2 charged Higgs bosons $\left(\mathbf{H}^{ \pm}\right)$.

${ }^{3} m_{h}=125 \mathrm{GeV}$ interpreted as light CP even scalar of MSSM.
} 
Out of these while $\phi \rightarrow b \bar{b}$ and $\phi \rightarrow \tau \bar{\tau}$ searches are more sensitive in the high $\tan \beta(>2)$ regime, the searches $\mathbf{H} \rightarrow \mathrm{hh}(\mathbf{A} \rightarrow \mathrm{Zh})$ have significant branching fractions in the high SUSY breaking scale $\left(M_{S U S Y}>1 \mathrm{TeV}\right)$ and low $\tan \beta$ region of the MSSM parameter space within mass range 260 (220) $\mathrm{GeV}$ to $350 \mathrm{GeV}$ where the lower bound is motivated by the kinematic thresholds for the daughters $\mathrm{hh}(\mathrm{Zh})$ to be produced on-shell and the upper bound is due to the dominance of branching ratio of light Higgs bosons to decay to top quarks. Besides, the $\mathbf{H} \rightarrow \mathrm{hh}$ decay mode is also present in $2 \mathrm{HDM}$ model. In the alignment/decoupling regime (when $\cos (\beta-\alpha)<<1$ where $\alpha$ is the mixing angle between two neutral scalar fields), the lightest Higgs becomes exactly Standard Model like and the $\mathbf{H} \rightarrow \mathrm{hh}$ and $\mathbf{A} \rightarrow \mathrm{Zh}$ couplings vanish. However, in the MSSM they don't vanish due to large radiative corrections and in the decoupling regime, for large mass of $\mathbf{H}$, the $\mathbf{H} \rightarrow t \bar{t}$ channel opens up and dominates.

\section{Event and Object reconstruction}

CMS uses a Particle Flow (PF) algorithm [4] for global event reconstruction using full granularity of all the subdetectors. This algorithm reconstructs isolated leptons (Muons) from the event followed by charged hadrons, electrons, photons and neutral hadrons (which are all clustered into Jets using the anti-kT algorithm [5]). $\tau$-leptons further decay either leptonically (into light leptons along with 2 neutrinos) or hadronically (into a narrow jet of charged hadrons, 1 neutrino and/or neutral pions). Consequently, $\tau$-leptons need to be identified from their decay products. While leptonic $\tau$ decay identification is similar to prompt leptons ${ }^{4}$, hadronic $\tau$ decay identification uses a dedicated algorithm. The hadronic $\tau$-lepton identification efficiency at CMS is $60 \%$ while the probability of a quark/light lepton to fake a $\tau$-lepton remains within $0.1 \%$ to $1 \%$ [6].

To identify jets coming from b-quarks, a likelihood based algorithm is used which combines secondary vertex and track-based lifetime information to compute a score that is directly proportional to the probability that the jet has come from a b-quark. It should be noted that identification of b-quarks is subject to resolution effects. The CMS b-quark tagging algorithm has an efficiency of $70 \%$. Light jets (u/d/s quarks) are misidentified as a b-quark approximately $1 \%$ of times [7].

\section{Analysis strategy \& Event categorization}

In the $\phi \rightarrow \tau \bar{\tau}$ search, events are categorized into "b-tag" and "no b-tag" categories depending on whether they have at least one or no b-tagged jets $\left(p_{T}>20 \mathrm{GeV}\right.$, where $p_{T}$ is the transverse momentum of jet) in them followed by requirement of two opposite-sign taus. Channels with hadronically-decaying taus in the final state were binned in hadronic tau $p_{T}$ and were required to satisfy multi-variate isolation criteria for enhanced analysis sensitivity. In the $\phi \rightarrow b \bar{b}$ search, events were selected if they had at least 3 jets $\left(p_{T}^{\text {Jet } 1}>80 \mathrm{GeV}, p_{T}^{\text {Jet2 }}>70 \mathrm{GeV}, p_{T}^{\text {Jet } 3}>20\right.$ $\left.\mathrm{GeV},\left|\eta^{\text {Jet } 1 / \text { Jet } 2 / \text { Jet3 }}\right|<1.65,\left|\Delta \eta^{\text {Jet } 1, \text { Jet } 2}\right| \leq 1.4\right)$ where, pseudo-rapidity $(\eta)$ equals $-\ln [|\tan (\theta / 2)|]$ with $\theta$ being measured w.r.t beam line. The 2 leading jets should satisfy track-based online btagging selections. These jets were also required to be pair-wise separated by $\Delta R>1$, where $\Delta R$ $=\sqrt{\delta \phi^{2}+\delta \eta^{2}}$ with $\delta \phi(\delta \eta)$ being the difference in the azimuthal angles (pseudo-rapidities) of

\footnotetext{
${ }^{4}$ Leptons coming directly from hard interaction vertex e.g W $\rightarrow 1 v / \mathrm{Z} \rightarrow 11$, where $1=\mathrm{e} / \mu$.
} 
the jets. The selected events were then divided into "triple b-tag" and "double b-tag" categories depending on the number of jets: 3 (2) passing the offline b-tagging criteria which were then used for signal extraction and background evaluation respectively.

Events were selected in the $\mathbf{H} \rightarrow$ hh search if they have at least 2 jets which are then categorized (depending on the number of b-tagged jets in them) into 0-tags, 1-tag, and 2-tags categories. Mass window cuts were applied to the di-tau and the b-quark systems to enhance the signal purity and a kinematic fit performed where energies of the b-jets and taus is varied within their respective resolutions subject to the $125 \mathrm{GeV}$ mass constraint.

In the $\mathbf{A} \rightarrow Z \mathrm{Zh}$ search, events are first selected if they have well reconstructed pair of oppposite sign light leptons consistent with the $\mathrm{Z}$ mass window. For the $h \rightarrow \tau \tau$ case, a channel dependent cut is applied on the scalar $p_{T}$ sum of the tau candidates to reduce backgrounds. Finally, for the $h \rightarrow b \bar{b}$ case, at least one tight b-tagged Jet and one loose b-tagged Jet is required in the event.

\section{Backgrounds}

Important backgrounds for the $\phi \rightarrow \tau \bar{\tau}, \phi \rightarrow b \bar{b}$ and $\mathbf{H} \rightarrow \mathrm{hh}$ searches are:

1. Drell-Yan $(Z \rightarrow \tau \tau)+\mathbf{J e t s}:$ This is the dominant irreducible background in $\mu \tau_{h}, e \tau_{h}, \tau_{h} \tau_{h}$ and $e \mu$ final states (where e $/ \mu / \tau_{h}$ denotes decay of tau to electron/muon/hadrons) in the $\phi \rightarrow \tau \bar{\tau}$ analysis and is also an important background in the $\mathbf{H} \rightarrow \mathrm{hh}$ search. It is estimated in shape from $Z \rightarrow \mu \mu$ events in data from which the muons are removed and replaced by tau leptons from simulation (Embedding).

2. W+Jets: This is an important reducible background in both $\phi \rightarrow \tau \bar{\tau}$ and $\mathbf{H} \rightarrow \mathrm{hh}$ searches and is caused by a jet $/ \mu$ being misidentified as a $\tau_{h}$. It is modelled in shape from simulation and in normalization from a high- $m_{T}$ region $\left(m_{T}>70 \mathrm{GeV}\right.$, where $m_{T}$ is the transverse mass between muon and missing transverse energy) in data in the $\mu \tau_{h}$ final state which is rich in $\mathrm{W}+\mathrm{Jets}$ events.

3. QCD MultiJets: This is the dominant background in the $\tau_{h} \tau_{h}$ channel in both $\phi \rightarrow \tau \bar{\tau}$ and $\mathbf{H} \rightarrow$ hh analysis and is estimated using a data-driven method from control regions defined by di-tau charge and isolation. It is also the main background in $\phi \rightarrow b \bar{b}$ search when at least two energetic jets actually contain B hadrons, and a third jet passes the b-tagging selection as a result of mistagging. Since this type of background cannot be accurately predicted by simulation, it is estimated from data using dedicated control samples.

4. $t \bar{t}+\mathbf{J e t s :}$ This is the main irreducible background in the $e \mu$ channel in $\phi \rightarrow \tau \bar{\tau}$ and in $\mathbf{H} \rightarrow \mathrm{hh}$ analysis. It is estimated in shape from simulation and in yield from a region in data in $e \mu$ channel which is rich in $t \bar{t}$ events.

5. Drell-Yan $(Z \rightarrow l l)+\mathbf{J e t s}$ : This background primarily arises in in $\phi \rightarrow \tau \bar{\tau}$ and $\mathbf{H} \rightarrow$ hh searches from $\mathrm{e} \rightarrow \tau_{h}$ and $\mu \rightarrow \tau_{h}$ fakes and is estimated from data. 
6. WW/WZ/ZZ+Jets: This is a small background in both $\phi \rightarrow \tau \bar{\tau}$ and $\mathbf{H} \rightarrow$ hh searches. It is estimated from simulation.

7. Single top: This is a small background and is estimated from simulation.

8. SM Higgs background: This is basically the sum of all processes involving decays of SM Higgs boson which pass the event selections. It is also estimated from simulation.

Similarly, the main backgrounds for the $\mathbf{A} \rightarrow \mathrm{Zh}$ search are:

1. ZZ di-boson production: This is the dominant irreducible background and is estimated from NNLO simulation.

2. Reducible backgrounds: This is mainly due to $Z+$ jets and $W Z+j e t s$ and is estimated in yield using fake rate method and in shape from same-sign sideband region of the 2 taus.

3. Rare backgrounds: These are due to processes like: $Z^{*} \rightarrow \mathrm{Zh} \rightarrow l \bar{l} \tau \bar{\tau}(b \bar{b}), Z^{*} \rightarrow \mathrm{Zh} \rightarrow \mathrm{ZWW} \rightarrow l \bar{l} \tau \bar{\tau}(b \bar{b})$, tri-boson (WWZ/WZZ/ZZZ) production and $t \bar{t} \mathrm{Z}$ (where $\mathrm{Z} \rightarrow l \bar{l}$ and $t / \bar{t} \rightarrow e / \mu / \tau$ ) and are estimated from simulation.

\section{Results}

In all these searches, no signal was observed. Hence exclusion limits were set on the "crosssection $\times$ branching ratio" for BSM Higgs production via a binned maximum likelihood fit to the data. These limits were computed via two different methods:

1. Model Independent method: Where dependence of both cross-section and branching ratio on the model parameters of the theory are ignored.

2. Model Dependent method: Where limits are set on regions of model parameter space defined by setting the model parameters to specific (benchmark) values based on latest theoretical and/or experimental constraints prior to the search.

Some of these limits are shown in Figures 1 and 2.

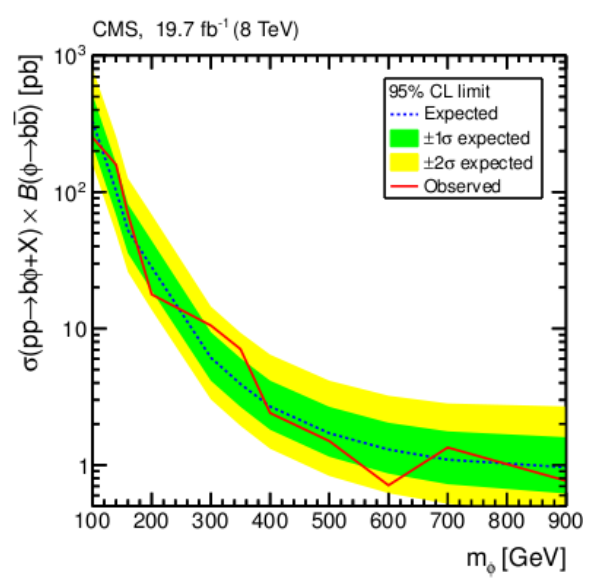

(a) Model independent limits for $\phi \rightarrow b \bar{b}$ search

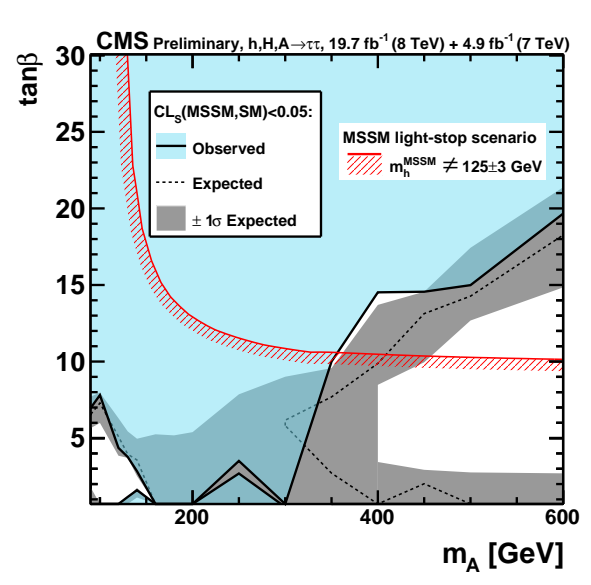

(b) Model dependent limits for $\phi \rightarrow \tau \bar{\tau}$ search

Figure 1: Exclusion limits at $95 \%$ Confidence level for $\phi \rightarrow b \bar{b}$ (left) and $\phi \rightarrow \tau \bar{\tau}$ (right) searches. 


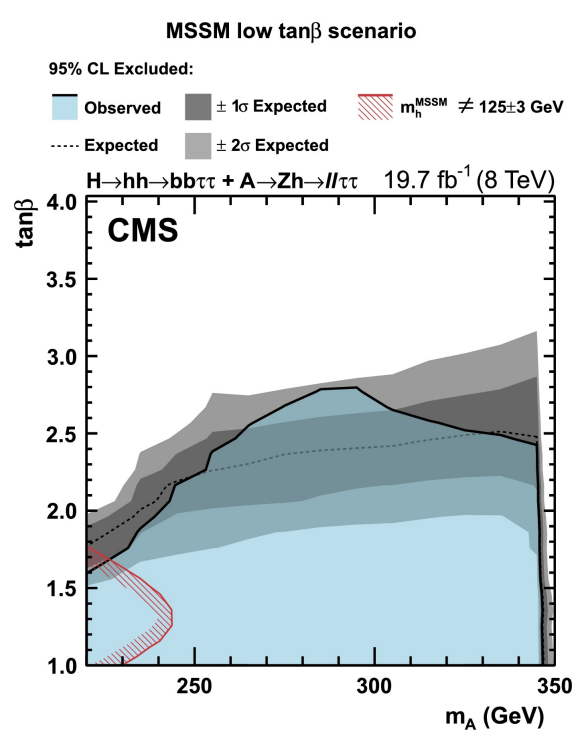

(a) Limits for MSSM

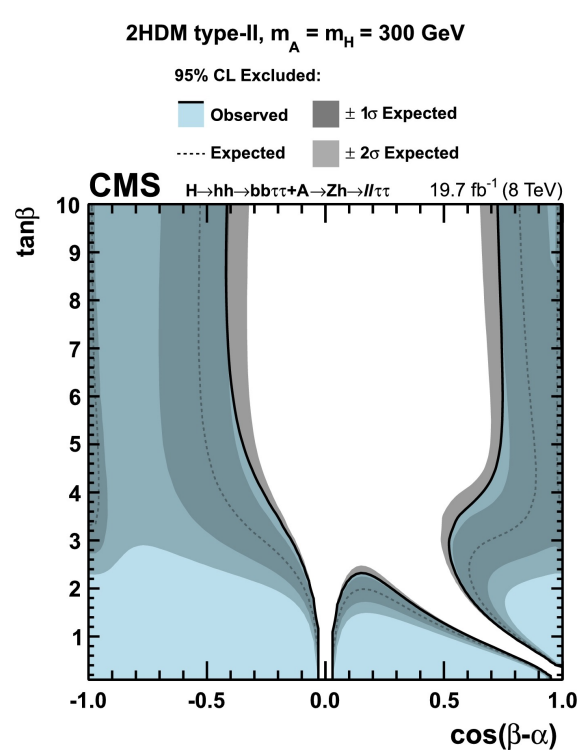

(b) Limits for $2 \mathrm{HDM}$

Figure 2: Model dependent exclusion limits at $95 \%$ confidence level for combination of $\mathbf{H} \rightarrow \mathrm{hh} \rightarrow b \bar{b} \tau \tau$ and $\mathbf{A} \rightarrow \mathrm{Zh} \rightarrow l \bar{l} \tau \tau$ searches.

The model independent limits exclude BSM higgs bosons (of masses upto $\sim 1 \mathrm{TeV}$ ) with production cross-sections upto $1 \mathrm{pb}$. In the model dependent case, large areas of MSSM and 2HDM parameter space were excluded in the proposed benchmark scenarios [12]. This exclusion was either theoretical on the basis of incompatibility to the observation of the $125 \mathrm{GeV}$ Higgs boson (within a theoretical uncertainity of $\pm 3 \mathrm{GeV}$ ) or experimental due to the results of the direct searches.

\section{References}

[1] CMS collaboration, The CMS experiment at the CERN LHC, Journal of Instrumentation, 3(08):S08004, 2008.

[2] CMS collaboration, Observation of a new boson at a mass of $125 \mathrm{GeV}$ with the CMS experiment at the LHC, Physics Letters B, 716:30-61, 2012, arXiv:1207.7235.

[3] CMS collaboration, Precise determination of the mass of the Higgs boson and tests of compatibility of its couplings with the standard model predictions using proton collisions at 7 and $8 \mathrm{TeV}$, The European Physical Journal C, 75(5):1-50, 2015, arXiv:1412.8662.

[4] CMS collaboration, Particle-Flow Event Reconstruction in CMS and Performance for Jets, Taus, and Missing $E_{T}, C M S$ PAS PFT-09-001 (2009), https://cds.cern.ch/record/1194487

[5] M. Cacciari and Gavin P. Salam, The anti-kt jet clustering algorithm, Journal of High Energy Physics, 2008(04):063, 2008, arXiv:0802.1189v2.

[6] CMS collaboration, Reconstruction and identification of tau lepton decays to hadrons and tau neutrino at CMS, Journal of Instrumentation, 11(01):P01019, 2016, arXiv:1510.07488. 
[7] CMS collaboration, Identification of b-quark jets with the CMS experiment, Journal of Instrumentation, 8:4013P, 2013, arXiv:1211.4462.

[8] CMS collaboration, Search for additional neutral Higgs bosons decaying to a pair of tau leptons in pp collisions at $\sqrt{s}=7$ and $8 \mathrm{TeV}$, CMS-PAS-HIG-14-029, http://cds.cern.ch/record/2041463.

[9] CMS collaboration, Search for neutral MSSM Higgs bosons decaying into a pair of bottom quarks, Journal of High Energy Physics, 2015(11):1-43, 2015, arXiv:1506.08329.

[10] CMS collaboration, Searches for a heavy scalar boson $\mathrm{H}$ decaying to a pair of $125 \mathrm{GeV}$ Higgs bosons hh or for a heavy pseudoscalar boson A decaying to $\mathrm{Zh}$, in the final states with $\mathrm{h}$ to tau tau, Physics Letters B, 755:217-244, 2015, arXiv:1510.01181.

[11] CMS collaboration, Search for a pseudoscalar boson decaying into a $Z$ boson and the $125 \mathrm{GeV}$ Higgs boson in $l^{+} l^{-} b \bar{b}$ final states, Physics Letters B, 748:221-243, 2015, arXiv:1504.04710.

[12] M. S. Carena, S. Heinemeyer, C. E. M. Wagner and G. Weiglein, MSSM Higgs boson searches at the Tevatron and the LHC: Impact of different benchmark scenarios, The European Physical Journal C, 73(9):1-20, 2013, arXiv:hep-ph/0511023. 